\title{
Linear Distribution System State Estimation with Integration of DG
}

\author{
S. Bindu ${ }^{1} \cdot$ S. Ushakumari ${ }^{2} \cdot$ J. S. Savier ${ }^{3}$
}

Received: 29 May 2020 / Accepted: 22 December 2020 / Published online: 9 January 2021

(C) The Author(s), under exclusive licence to Springer Nature Singapore Pte Ltd. part of Springer Nature 2021

\begin{abstract}
Monitoring of a power system is essential for maintaining it in a secure operating condition. Electric distribution management system (DMS) should possess a monitoring mechanism addressing the entire operating conditions of the system. Distribution System State Estimation (DSSE) is an integral building block of the DMS. Because of new technologies integrated into the power distribution system, new opportunities, problems and challenges related to control and management are being explored. To make efficient and reliable control actions, an accurate knowledge of the system states is required. This paper presents Linear State Estimator based DSSE which can handle the challenges mentioned above. The estimator studied and reported in the paper allows to include synchronized phasor measurements provided by Phasor Measurement Units (PMUs). With the increasing integration of DG in distribution system, the state estimation scheme needs be reworked to address the complexities introduced by power exchange of DG. To address this, Linear State Estimation (LSE) is reformulated and its performance is analysed with DG integrated in the sample network under study.
\end{abstract}

Keywords Power system · Distribution system state estimation · Linear state estimator · Distributed generation · Phasor measurement unit

\section{Introduction}

Monitoring in real time is highly essential for implementing real time control and protection functions of a distribution electric network. Available measurements are to be used properly so that the best approximate of the system state which fits the measurements can be obtained. DSSE is treated as key component of DMS. Transmission System State Estimation (TSSE) is well developed over the years. Due to inherent and fundamental differences in transmission and distribution systems, TSSE cannot be directly applied for functional requirements of DSSE.

The unique characteristics of distribution system include load unbalances, high $\mathrm{R} / \mathrm{X}$ ratios and less observability due to inadequate measurements in real time. The main limitation is the lesser number of measurements due to various practical

S. Bindu

bindus@cet.ac.in

1 Government Engineering College, BartonHill, Thiruvananthapuram, India

2 TREST Research Park, Thiruvananthapuram, India

3 College of Engineering Trivandrum, Thiruvananthapuram, India and economic reasons. Transmission systems have sufficient measurements and many of them are sufficiently redundant making systems always visible.

In the case of distribution systems, real time measurements are less and therefore the system is not observable. Some data, known as pseudo measurements, are used in order make the system observable. The use of PMUs in distribution systems give accurate information about voltage magnitude and phase angle which in turn can improve the estimation accuracy of DSSE. In a modern-day distribution grid, the performance requirements of DSSE are becoming stringent. This is due to the challenges introduced by integration of DG and use of advanced technologies. Ref. [1] summarizes the major obstacles and challenges associated with DSSE.

Various methods of formulating the mathematical problem, application of pseudo measurements, placement of metering instruments etc. are investigated in [2]. The conventional method and modern data-driven and probabilistic techniques are reviewed. The role and significance of pseudo measurements due to limited real-time measurements and network observability is emphasized in [3-7]. A hybrid state estimator based on SCADA and using PMU measurements are proposed in [8], which results in an improved and robust estimation. 
Sizing and placement of Distributed Generation (DG) for improved performance of a distribution system is proposed in [9]. This paper also proposes techniques used to improve adaptive capability of the network to handle extreme events. Ref [10] explains optimal placement of DG in radial distribution network using evolutionary algorithms like Genetic Algorithm and Particle Swam Optimisation. A multi-objective performance index-based sizing and placement of DG in distribution systems is studied in [11]. Load is considered to be varying by considering the effect of industrial, residential and commercial loads. The issues in the allocation of DG is presented along with an analytical method for the allocation of DG in radial distribution systems in [12].

Various aspects of State Estimation (SE) of a distribution grid, which is observable, using Weighted Least Square and Extended Kalman Filter are investigated in [13]. The main disadvantage of these methods is that they need some prior knowledge about the state of the system for a satisfactory performance.

Methods like Branch-Current-based SE (BCSE) take information from network structure and the nature of the measurements. An efficient and robust branch current based DSSE with minimum storage requirements is presented in [14]. The method of including phasor measurement data in post processing mode in transmission systems is discussed in [15] whereas [16] presents a DSSE method which uses only few measurements and gives a SE with improved accuracy. Singular Value Decomposition (SVD), Principal Component Analysis (PCA) algorithms for data compression in SE and BCSE method to find the system states for the distribution network are addressed in [17].

Various types of estimators are explained in [18] where the importance of real time monitoring, accurate estimation of network topology and timely identification of the topology changes etc. are emphasized. SE with microgrids is studied in [19]. A recursive Bayesian estimator is developed and reported in [20]. A normalized residual test for detection of network topology change is studied in [21]. The Branch Current based SE (BCSE) and the challenges in DSSE are investigated in [22]. The SE algorithm explained in [23] refers to a compensation method to cope with the low redundancy of measurement sets. Even though this method prevents the illconditioning of the gain matrix, the solution obtained suffers from low accuracy.

Ref. [24] addresses the problem of minimum number of measurements and discusses semidefinite programming technique for DSSE to deal with this problem. Augmentation of pseudo measurements using ANN based forecasting is used for tackling challenges due to limited measurements in distribution system as reported in [25]. The historical data used for training the ANN is highly critical as erroneous forecasted values will eventually result in inaccurate SE. It is also reported that such algorithms bring in the challenge of increased execution time.

Weighted Least Square (WLS) technique is widely used in DSSE. But the instability issue, sensitivity to initial point etc. are the problems in this technique. Several methods are proposed to tackle the problems associated with the WLS method. As a prospective and promising solution, development of a LSE method and its performance are addressed in this paper. The method accounts for PMU measured data and measurements by line current sensors. To address the issues related to insufficient number of measurements in a distribution network, pseudo measurements are introduced using the estimated values from a linear load flow. The linear load flow is opted since it is easy to implement and accurate. A linearization method based on the Taylor's series approximation is adopted in the load flow. These measurements, along with the available actual measurements are used in LSE which eventually brings out the system states. LSE is non iterative and overcomes the issues associated with WLS method. From the results, it can be seen that the LSE can be a good choice when the electric network has a limited number of actual measurements and there are pseudo-measurements which are generally inaccurate as compared to the actual measurements. The performance of the SE method proposed is also tested with DG integrated at specific nodes of the sample network under study.

LSE methodology proposed is covered in detail in "Linear Distribution State Estimation (LSE)" section of this paper. The sizing and placement of DG and LSE methodology integrating DG are discussed in "LSE in the Presence of DG" section. In "Results" section, the simulation results of the proposed method on an IEEE 33 bus is discussed. Fifth section gives the conclusions of the work carried out.

\section{Linear Distribution State Estimation (LSE)}

To find out the true values in the network, a linear load flow for three-phase power distribution systems is used. ZIP model of the loads are considered along with balanced and unbalanced loads. The method is very simple and accurate. The problem of high $\mathrm{R} / \mathrm{X}$ ratio is addressed by using a linear approximation.

The admittance matrix relating nodal voltages and currents is as follows:

$$
\left(\begin{array}{c}
I_{A} \\
I_{B}
\end{array}\right)=\left(\begin{array}{ll}
Y_{A A} & Y_{A B} \\
Y_{B A} & Y_{B B}
\end{array}\right)\left(\begin{array}{l}
V_{A} \\
V_{B}
\end{array}\right)
$$

The current in each node is related to the voltage as:

$$
I_{k}=\frac{S_{P k}^{*}}{V_{k}^{*}}+m \cdot S_{I k}^{*}+m^{2} \cdot S_{Z k}^{*} \cdot V_{k}
$$


where $\mathrm{m}=1 / \mathrm{V}_{\text {nominal }}$

A linear approximation for $\mathrm{V}$ is considered as

$\mathrm{V}=\mathrm{f}(\Delta \mathrm{V})=(1-\Delta \mathrm{V})$

when $\Delta \mathrm{V}$ is very small. A linear form is obtained as in (3) by evaluating Taylor series around zero where higher order terms are neglected and $\mathrm{V}=(1-\Delta \mathrm{V})$ is used.

$\frac{1}{V}=\frac{1}{1-\Delta V} \approx 1+\Delta V=2-V$

An expression for the nodal current (2) in linear form is obtained as below

$M+N \cdot V^{*}{ }_{B}+Q \cdot V_{B}=0$

with,

$$
\begin{aligned}
& M=Y_{B A} \cdot V_{A}-2 m \cdot S_{P B}^{*}-m \cdot S_{I B}^{*} \\
& N=m^{2} \cdot \operatorname{diag}\left(S_{P B}^{*}\right) \\
& Q=Y_{B B}-m^{2} \cdot S^{*}{ }_{Z B}
\end{aligned}
$$

(4) can be solved using rectangular representation as follows:

$$
\left(\begin{array}{c}
-M_{r} \\
-M_{i}
\end{array}\right)=\left(\begin{array}{cc}
N_{r}+Q_{r} & N_{i}-Q_{i} \\
N_{i}+Q_{i} & -N_{r}+Q_{r}
\end{array}\right) \cdot\left(\begin{array}{c}
V_{r} \\
V_{i}
\end{array}\right)
$$

where $r$ and $i$ indicate the real and imaginary parts respectively of the parameters in rectangular form.

Equation (8) is used to calculate the true values of voltage. The magnitude of true value of current and power are derived from this. Since the real measurements are limited in number, pseudo measurements are substituted. The pseudo measurements are derived from true values by adding a random error to the actual values. These values are then used in LSE.

The measurement equation for weighted least square estimation is,

$z=h_{1}(x)+e$

where $x$ is the state vector and the relationship between measurements and state variables is denoted by $h_{1}(x) . e$ is the vector of measurement errors having a Gaussian distribution with zero mean and covariance $R$.

The WLS problem is then defined as,

$$
\operatorname{Min} J(x)=\left[z-h_{1}(x)\right]^{T} R^{-1}\left[z-h_{1}(x)\right]
$$

Since $h_{1}(x)$ is a non-linear function, iterative techniques are required to solve (10). But these methods greatly depend on the initial guess and are prone to divergence, particularly when the errors of various measurements vary significantly. Moreover, loads in the distribution system, do not follow a specific pattern. This also is considered as a difficulty in applying WLS in DSSE problems.

These problems are handled in LSE. The solution of LSE can be obtained without iterations since the measurement functions are linear.

The measurement functions include node voltage, current and complex power.

The node voltage is defined as $V=a+i b$ where $a$ is the vector defining the real part and $b$ is the vector defining the imaginary part of the node voltage. Some of the node voltages are directly measured by PMUs which are included in SE to enhance the performance of state estimation.

Line current sensors are used to measure the current vector. The measurement model for line current measurement is,

$I_{i j}=\left(V_{i}-V_{j}\right) Y_{i j}$

The complex current vector is represented as

$\operatorname{Re}\left\{\mathrm{I}_{\mathrm{ij}}\right\}=\left(\mathrm{a}_{\mathrm{i}}-\mathrm{a}_{\mathrm{j}}\right) \mathrm{G}_{\mathrm{ij}}-\left(\mathrm{b}_{\mathrm{i}}-\mathrm{b}_{\mathrm{j}}\right) \mathrm{B}_{\mathrm{ij}}$

$\operatorname{Im}\left\{I_{i j}\right\}=\left(a_{i}-a_{j}\right) B_{i j}+\left(b_{i}-b_{j}\right) G_{i j}$

where $G_{i j}$ and $B_{i j}$ are the real part and imaginary part of $Y_{i j}$, the admittance of line connecting the $i^{t h}$ and $j^{\text {th }}$ nodes in the network.

Load measurements are usually obtained through load forecasting or historical data regarding consumption.

The equation for power injection is,

$V_{i} I^{*}{ }_{i}=S_{i}$

where $I_{i}^{*}$ is the conjugate of the current at node $i$ and $S_{i}$ is the apparent power at node $i$ which is a non-linear function.

Taylor series based expansion is used in this work to make the above equation linear.

Let the voltage at $i^{\text {th }}$ bus is denoted in per unit as,

$V_{i}=1-\Delta V_{i}$

By Taylor series expansion and approximation, it can be written from (3) that,

$\frac{1}{V_{i}}=2-V_{i}$

The error introduced by this approximation is very small. Substituting (15) in (13)

$I^{*}{ }_{i}=S_{i}\left(2--V_{i}\right)$

Re writing $I_{i}$ based on the node voltages, (16) can be written as

$\sum_{j \in N_{i}}\left(V_{i}-V_{j}\right) Y_{i j}=S_{i}^{*}\left(2-V_{i}^{*}\right)$

where $N i$ is the set of all nodes linked to the node $i$. 
By re-organising (17) the complex power can be separated out as below:

$$
\begin{aligned}
\sum_{j \in N_{i}} & {\left[\left(a_{i}-a_{j}\right) G_{i j}-\left(b_{i}-b_{j}\right) B_{i j}\right]+a_{i} P_{i}-b_{i} Q_{i} } \\
& =2 P_{i} \sum_{j \in N_{i}}\left[\left(a_{i}-a_{j}\right) B_{i j}+\left(b_{i}-b_{j}\right) G_{i j}\right]-a_{i} Q_{i}-b_{i} P_{i}=-2 Q_{i}
\end{aligned}
$$

Thus, considering all the measurement functions - node voltages, line currents and load, the system measurement function will be

$\left[\begin{array}{c}h_{1}{ }^{V} \\ h_{1}{ }^{I} \\ h_{1}{ }^{S}\end{array}\right] x=\left[\begin{array}{c}z^{V} \\ z^{I} \\ z^{S}\end{array}\right]$

which is linear.

The state estimation can now be solved using (20)

$x=\left[H_{1}^{T} W H_{1}\right]^{-1} H_{1}^{T} W z$

where $\mathrm{H}_{1}$ is the Jacobian matrix and $W$ is the inverse of the covariance matrix and the errors are having a Gaussian distribution with zero mean.

\section{LSE in the Presence of DG}

\section{Placement and Sizing of DG}

DGs are an integral part in any modern day power distribution network. Improvement of voltage profile in electric distribution network is an important additional motive for integration of DG. Placement of DG improves the voltage profile and the stability of the system. The SE method must consider the interconnection of DG in the network and the algorithm needs to be modified accordingly. Placement and sizing of DG in electric distribution systems is decided considering the influence of the inclusion on the operating parameters of the network. By optimally placing DG in the electric distribution network results in the decrease of the total power loss and improvement of voltage profile of the system. Optimal placement of DG is based on an index called as Power Stability Index (PSI) [12]. PSI of bus $m$ is expressed as below (21)

$P S I=\frac{4 R_{m n}\left(P_{m}-P_{n}\right)}{\left[\left|V_{m}\right| \cos \left(\theta_{m}-\theta_{n}\right)\right]^{2}}$

where $\mathrm{R}_{\mathrm{mn}}$ is the resistance of the line connecting $m^{\text {th }}$ and $n^{\text {th }}$ buses, $P_{m}$ and $P_{n}$ are real power at bus $m$ and bus $n$ respectively, Magnitude of voltage at $\mathrm{m}^{\text {th }}$ bus is denoted as $\mathrm{V}_{\mathrm{m}} \cdot \theta_{\mathrm{m}}$ and $\theta_{\mathrm{n}}$ are the angles at $\mathrm{m}^{\text {th }}$ and $\mathrm{n}^{\text {th }}$ bus respectively. PSI is calculated for every bus in a network. The indices are then arranged in descending order. DG can be placed in the bus which is having highest PSI index for maximum advantage.

The sizing of the DG shall be compatible with the rating of the system. The power delivered from the DG should be absorbed within the pre-defined distribution area. There is an optimum size of the DG in a bus, at which the losses in the system is a minimum. If the DG size is not the optimum value, the power flow limit exceeds and it will create losses. Thus in order to keep the losses to a minimum value, the DG size is fixed to an optimum value. Analytical approach is used for optimum DG size selection [11]. The method is easy to implement and computationally efficient. It is based on the minimization of losses in the system.

The total loss in the system is,

$P_{\text {Loss }}=\sum_{i=1}^{n} \sum_{k=1}^{n}\left[A_{i k}\left(P_{i} P_{k}+Q_{i} Q_{k}\right)+B_{i k}\left(P_{k} Q_{i}-P_{i} Q_{k}\right)\right]$

Minimizing the power loss function with reference to the incremental power injected at node $i$,

$\frac{\delta P_{\text {Loss }}}{\delta P_{i}}=0$

Then the real power injected at $i^{\text {th }}$ bus is,

$P_{i}=\frac{1}{A_{i i}}\left[\sum_{k=1}^{n}\left[A_{i k} P_{k}-B_{i k} Q_{k}\right]\right]$

Fig. 1 Single line diagram of IEEE 33 bus radial distribution System

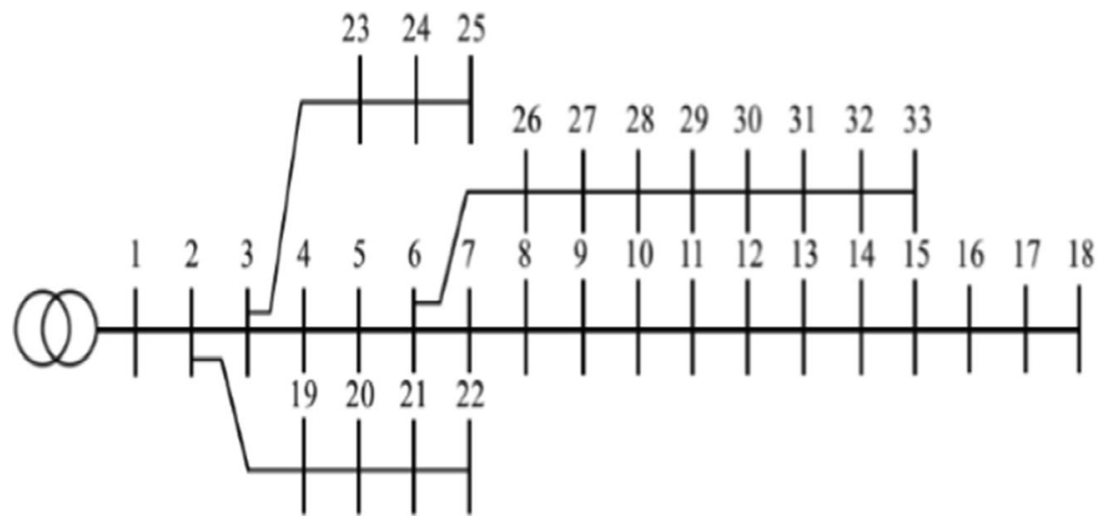


Fig. 2 Voltage magnitudes for a IEEE 33 bus radial distribution system estimated using LSE method

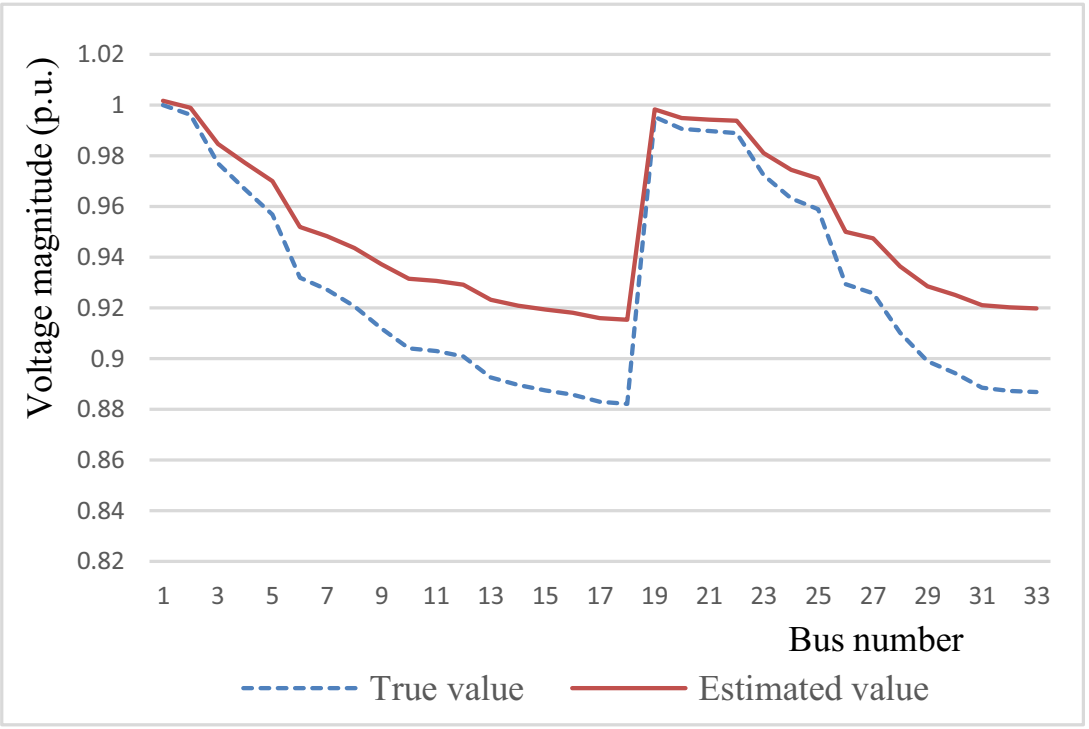

State Estimation with DG

$A_{i k}=\frac{R_{i k} \cos \left(\delta_{i}-\delta_{k}\right)}{V_{i} V_{k}}$

The performance of the new SE method is to be tested with inclusion of DG in the network. The position of DG interconnection and the rating of the resource are very critical when the operation of the overall network is considered. The optimum position for DG interconnection is found based on criteria decided by PSI values at different nodes. The sizing of the DG is carried out analytically as in "LSE in the Presence of DG" section and is included with the state estimation algorithm.

\section{Results}

The SE is performed on IEEE 33 bus (Fig. 1) radial distribution system. The true values are calculated using a linear load
Fig. 3 Voltage angles for the radial distribution System (IEEE 33 bus) estimated using LSE method

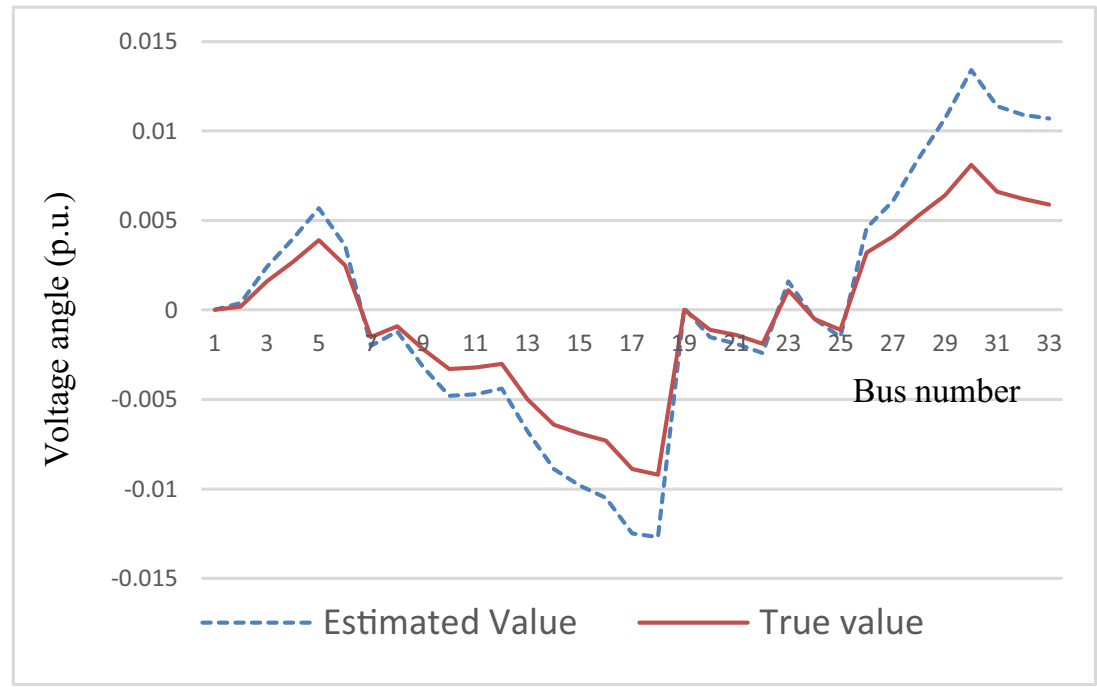


Fig. 4 Error in Voltage magnitudes for a 33 bus radial distribution system using LSE method and WLS method

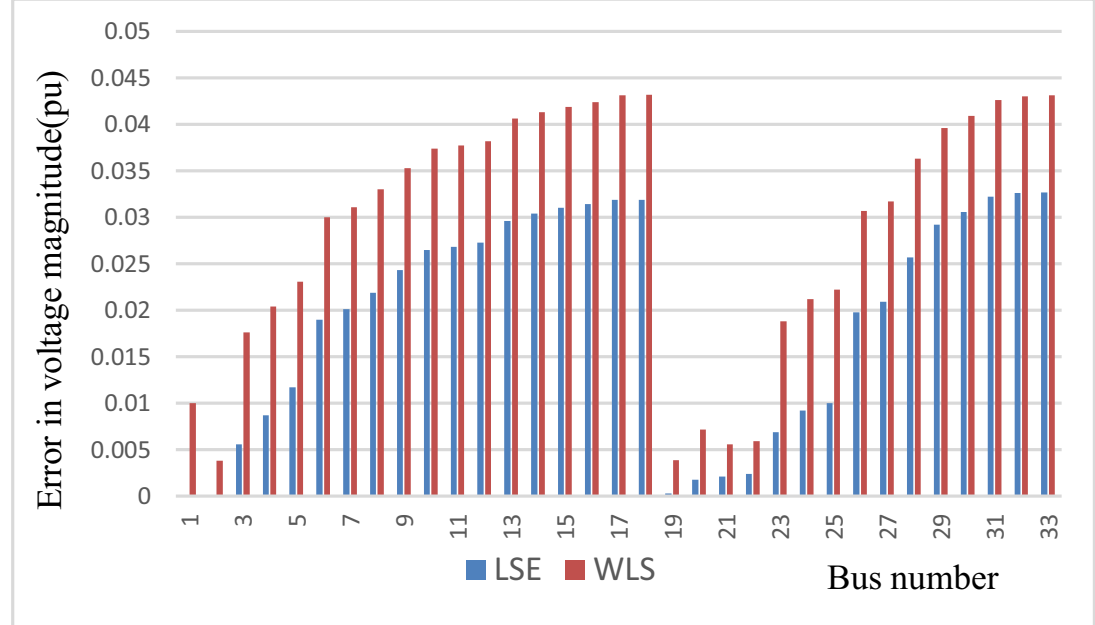

flow method. These values are then used for the calculation of pseudo measurements.

All measurements including pseudo measurements, PMU measurements and the line current measurements form the set of measurements for the SE.

The results of the newly proposed SE are shown in Figs. 2 and 3. The plots of the voltage magnitude are shown in Fig. 2. Estimated values are found to follow the true values of voltage magnitude. The errors are found to be comparatively less with that obtained from WLS method. Load flow analysis on the network provides the true values of various parameters of the distribution system under study. Figure 3 shows the angles associated with the voltage vector at various nodes of the system. Bus 1 is taken as the reference node for the estimation. In the case of voltage angles also, the estimated values closely follow the true values.

The errors in magnitude and angle of the bus voltage vector with reference to the true values are depicted in Figs. 4 and 5. Figures show that the errors are high in WLS method as compared to LSE method.

A detailed study of LSE incorporating DG is also carried out. DG is placed in the system based on the PSI values. The value of PSI is the highest in the eighth node and therefore the DG is placed in that node. This is the optimal place for placing the DG. The size of the DG is based on (23) which in turn depends on loss minimization in the system. The size of the DG is found to be $238.28 \mathrm{~kW}$ and is placed in node 8 . LSE is performed by placing the DG on node 8 . The voltage profile is shown in Fig. 6 and is found to improve with the integration of DG. The maximum improvement in voltage is found to be at node 18 and the losses are also reduced.

\section{Conclusion}

DSSE using LSE is proposed in this paper to overcome the drawbacks of the standard WLS method. The proposed method is suitable where true measurements are less in number which is compensated by pseudo measurements. The reported algorithm can also accommodate synchronized phasor measurement data to obtain more precise state estimates. The errors in the estimates with the proposed method are found to be less when compared to WLS technique. A methodology is evolved for
Fig. 5 Error in Voltage angles for a 33 bus radial distribution system using LSE method and WLS method

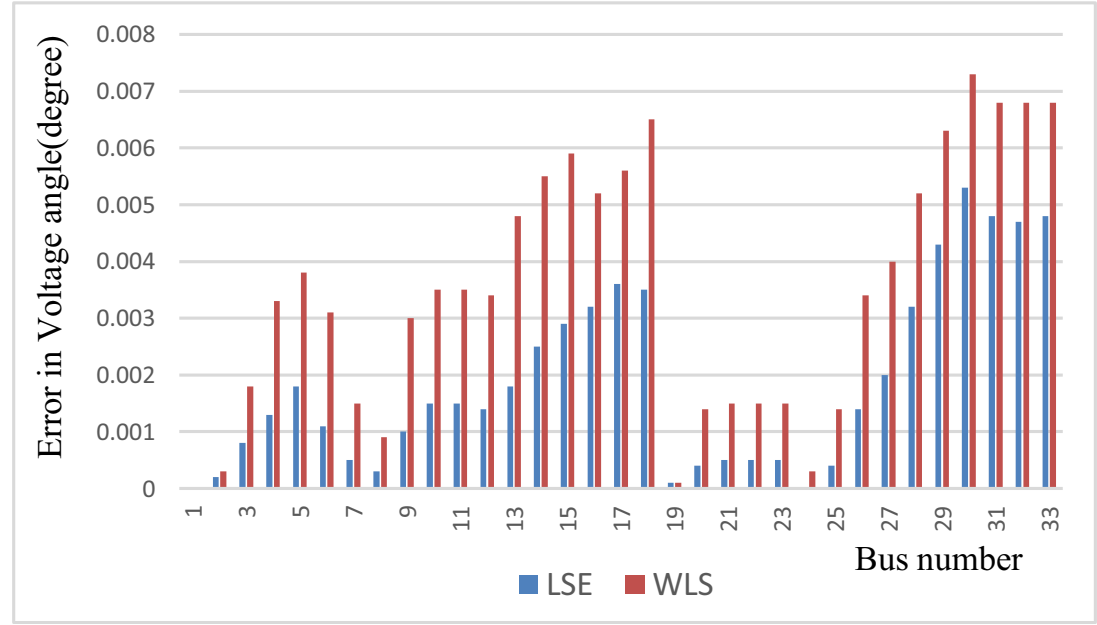


Fig. 6 Improvement in voltage profile with DG placement for a IEEE 33 bus radial distribution system

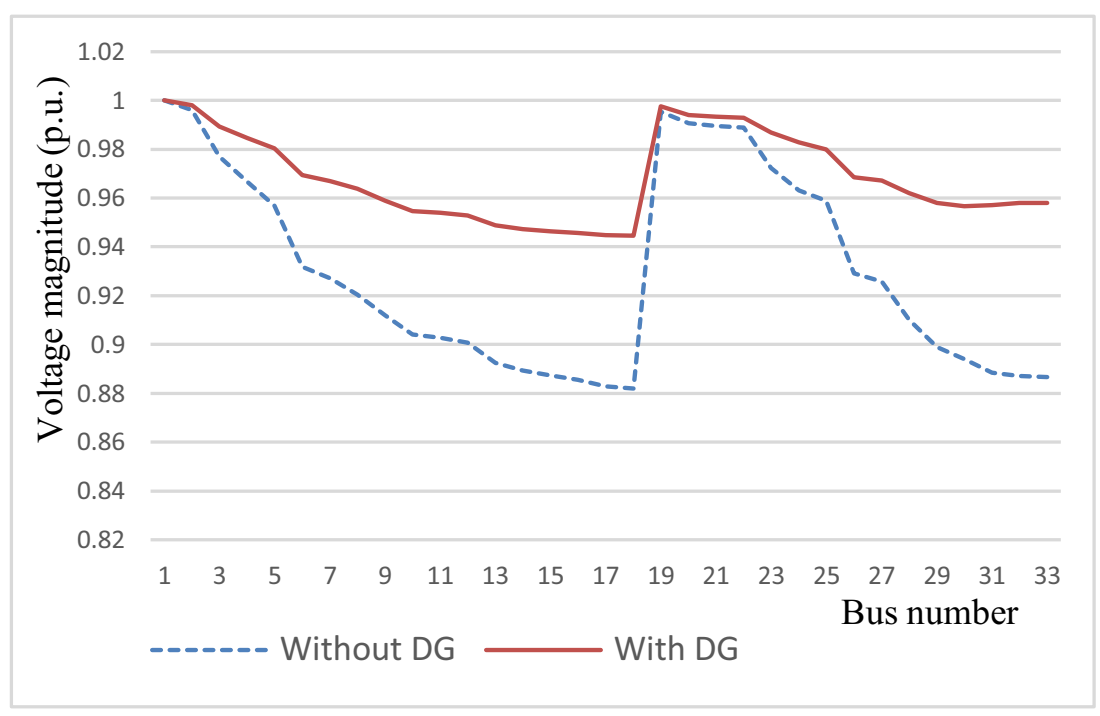

using LSE in DG interconnected distribution network and it is tested and verified. Voltage profile improvement is observed by placing the DG in the system. A comprehensive LSE algorithm is evolved, tested, verified and reported considering different aspects of a modern day electric distribution network.

\section{References}

1. Primadianto A, Lu C-N (2017) A review On DSSE. IEEE Tr Power Syst 32(5):3875-3883

2. Dehghanpour K, Wang Z, Wang J, Yuan Y, Bu F (2019) A survey on SE techniques and challenges in smart distribution systems. IEEE Tr.on Smart Grid 10(2):2312-2322

3. Baran ME, Kelley AW (1994) SE for real time monitoring of distribution system. IEEE Tr Power Syst 9(3):1601-1609

4. Lu CN, Teng JH, Liu WHE (1995) Distribution system state estimation. IEEE Tr Power Syst 10(1):229-240

5. Baran ME, Kelley AW (1995) A BCSE method for distribution systems. IEEE Tr. Power Syst 10(1):483-491

6. Ghosh AK, Lubkeman DL, Downey MJ, Jones RH (1997) DSSE using probabilistic approach. IEEE Tr. In Power Syst 12(1):45-51

7. Roytelman I, Shahidehpour SM (1993) SE for electric power distribution systems in quasi real time conditions. IEEE Tr. On Power Delivery 8(4):2009-2015

8. Kong X, Chen Y, Xu T, Wang C, Yong C, Lil P, Yu L (2018) A hybrid SE based on SCADA and PMU measurements for medium voltage distribution system. Journal of Applied Sciences

9. Sandhya K, Chatterjee K, Ghose T (Dec. 14-16, 2018) SE for DG penetrated adaptive distribution system during disaster. Proceedings of the National Power Systems Conference (NPSC)

10. Fathi M, Ghiasi M (June 2019) Optimal DG placement to find optimal voltage profile considering minimum DG investment cost in smart neighborhood. Smart Cities, MDPI Journal

11. Viswanatharao J, Praveena E, Srinivasarao R (2017) Placement and sizing of DG for distribution system by using a novel algorithm. IEEE Conference ICICCT

12. Chatterjee S, Roy BK (2015) An analytic method for allocation of DG in radial distribution system. IEEE Con INDICON
13. Gelagaev R, Vermeyen P, Driesen J (Oct.2008) SE in distribution grids. Int Conference on Harmonics and Quality of Power

14. Lin WM, Teng JH, Chen SJ (2001) A highly efficient algorithm in treating current measurements for the branch-current-based DSSE. IEEE Tr. on Power Delivery 16(3):433-439

15. Zhou M, Centeno VA, Thorp JS, Phadke AG (2006) An alternative for including phasor measurements in SE. IEEE Tr. on Power Syst 21(4):1930-1937

16. $\mathrm{Hu}$ Y, Kuh A, Yang T, Kavcic A (2011) A belief propagation based power distribution system state estimator. IEEE Computational Intelligence Magazine 6(3):36-46

17. Radhoush S, Shabaninia F, Lin J (Feb. 2018) DSSE with measurement data using different compression methods. IEEE Power and Energy Conference

18. Huang YF, Werner S, Huang J, Kashyap N, Gupta V (2012) SE in electric power grids: Meeting new challenges presented by the requirements of the future grid. IEEE Signal Proc Mag 29(5):33-43

19. Korres GN, Hatziargyriou ND, Katsikas PJ (2011) SE in multimicrogrids. Eur Tr Electr Power 21(2):1178-1199

20. Huang J, Gupta V, Huang YF (2012) Electric grid SE for distribution systems with microgrids. In Proc Inf Sci Syst (CISS), 46th Ann Conf: $1-6$

21. Korres G, Manousakis N (2012) A SE algorithm for monitoring topology changes in distribution systems. In Proc IEEE Power Energy Soc Gen Meeting:1-8

22. Wang H, Schulz N (Feb. 2004) A revised branch current-based DSSE algorithm and meter placement impact. IEEE Tr. on Power Syst 19(1):207-213

23. Gómez-Quiles C, Romero-Ramos E, de la Villa-Jaén A, GómezExpósito A (June 2020) Compensated load flow solutions for distribution system state estimation. Energies:1-13. https://doi.org/10. 3390/en13123100

24. Yao Y, Liu X, Zhao D, Li ZI (March 2019) DSSE-A semidefinite programming approach. IEEE Tr. on Smart Grid 10(4):4369-4378

25. Carcangiu S, Fanni A, Pegoraro PA, Sias G, Sulis S (February 2020) Forecasting-aided monitoring for the distribution system state estimation",WILEY:1-15. https://doi.org/10.1155/2020/ 4281219

Publisher's Note Springer Nature remains neutral with regard to jurisdictional claims in published maps and institutional affiliations. 